\title{
Population Pharmacokinetics and Dosing Optimization of Linezolid in Pediatric Patients
}

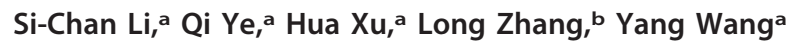 \\ aDepartment of Clinical Pharmacy, Wuhan Children's Hospital, Tongji Medical College, Huazhong University of Science \& Technology, Wuhan, China \\ bDepartment of Intensive Care Unit, Wuhan Children's Hospital, Tongji Medical College, Huazhong University of Science \& Technology, Wuhan, China
}

ABSTRACT Linezolid is a synthetic antibiotic very effective in the treatment of infections caused by Gram-positive pathogens. Although the clinical application of linezolid in children has increased progressively, data on linezolid pharmacokinetics in pediatric patients are very limited. The aim of this study was to develop a population pharmacokinetic model for linezolid in children and optimize the dosing strategy in order to improve therapeutic efficacy. We performed a prospective pharmacokinetic study of pediatric patients aged 0 to 12 years. The population pharmacokinetic model was developed using the NONMEM program. Goodness-of-fit plots, nonparametric bootstrap analysis, normalized prediction distribution errors, and a visual predictive check were employed to evaluate the final model. The dosing regimen was optimized based on the final model. The pharmacokinetic data from 112 pediatric patients ages 0.03 to 11.9 years were analyzed. The pharmacokinetics could best be described by a one-compartment model with first-order elimination along with body weight and the estimated glomerular filtration rate as significant covariates. Simulations demonstrated that the currently approved dosage of $10 \mathrm{mg} / \mathrm{kg}$ of body weight every $8 \mathrm{~h}$ (q8h) would lead to a high risk of underdosing for children in the presence of bacteria with MICs of $\geq 2 \mathrm{mg} /$ liter. To reach the pharmacokinetic target, an elevated dosage of 15 or $20 \mathrm{mg} / \mathrm{kg}$ q8h may be required for them. The population pharmacokinetics of linezolid were characterized in pediatric patients, and simulations provide an evidence-based approach for linezolid dosage individualization.

KEYWORDS children, dosing, linezolid, pharmacokinetics

inezolid is the first antibiotic in the oxazolidinone class and has been widely used -in the treatment of Gram-positive bacterial infections for more than a decade. By binding to the bacterial 23S rRNA of the 50S subunit and blocking the formation of the functional 70S initiation complex, linezolid inhibits protein synthesis and exhibits strong activity against resistant Streptococcus, Staphylococcus, and Enterococci species (1-3). Linezolid has been approved for pediatric use since 2002 in the United States, with indications for treatment including skin and skin structure infections, communityacquired or nosocomial pneumonia, and vancomycin-resistant Enterococcus-associated infections (including cases with concurrent bacteremia) $(4,5)$.

Linezolid is rapidly and extensively absorbed after oral dosing (tablet and suspension formulations), with a bioavailability of approximately $100 \%$. Therefore, the administration route of this agent can be switched from intravenous to oral in clinically stable patients without dose adjustment $(6,7)$. Linezolid is widely distributed to well-perfused tissues and body fluids and exhibits a low protein binding capacity of $31 \%$ (8). It is metabolized by nonenzymatic chemical oxidation mainly into two inactive carboxylic acid metabolites, PNU-142586 and PNU-142300. The dominant route of elimination is urinary excretion of the two main metabolites and the parent drug, accounting for approximately $80 \%$ of the linezolid dose $(9,10)$. Previous studies have suggested that
Citation Li S-C, Ye Q, Xu H, Zhang L, Wang Y. 2019. Population pharmacokinetics and dosing optimization of linezolid in pediatric patients. Antimicrob Agents Chemother 63:e02387-18 https://doi.org/10.1128/AAC.02387-18. Copyright $\odot 2019$ American Society for Microbiology. All Rights Reserved. Address correspondence to Yang Wang, cattop3211@qq.com.

Received 9 November 2018 Returned for modification 10 December 2018 Accepted 8 January 2019 Accepted manuscript posted online 14 January 2019

Published 27 March 2019 
TABLE 1 Demographic characteristics of the 112 children $^{a}$

\begin{tabular}{|c|c|c|c|}
\hline Characteristic & No. & Mean (SD) & Median (range) \\
\hline Patients & 112 & & \\
\hline \multicolumn{4}{|l|}{ Gender } \\
\hline Male & 65 & & \\
\hline Female & 47 & & \\
\hline Samples & 135 & & \\
\hline No. of samples per patient & & $1.2(0.4)$ & $1(1-3)$ \\
\hline Sampling time after the last dose (h) & & $5.8(2.6)$ & $7(0.5-8)$ \\
\hline Age $(y r)$ & & $2.9(3.4)$ & $1.6(0.03-11.9)$ \\
\hline WT (kg) & & $13.9(10.2)$ & $11.0(2.1-46.0)$ \\
\hline $\mathrm{Ht}(\mathrm{cm})$ & & $87.6(30.8)$ & $85.0(43.0-155.0)$ \\
\hline $\mathrm{BSA}\left(\mathrm{m}^{2}\right)$ & & $0.57(0.34)$ & $0.49(0.17-1.47)$ \\
\hline Linezolid dose (mg/kg/dose) & & $10.0(1.5)$ & $10.0(5.0-20.0)$ \\
\hline BUN (mmol/liter) & & $3.3(1.8)$ & $2.9(0.5-12.6)$ \\
\hline $\mathrm{SCR}(\mu \mathrm{mol} / \mathrm{liter})$ & & $25.0(8.7)$ & $23.8(9.6-55.2)$ \\
\hline UA ( $\mu \mathrm{mol} /$ liter) & & $201.9(81.9)$ & $190.3(41.8-504.8)$ \\
\hline Cys-C (mg/liter) & & $0.9(0.3)$ & $0.9(0.4-1.9)$ \\
\hline eGFR (ml/min.1.73 m²) & & $136.5(50.0)$ & $132.6(45.1-338.1)$ \\
\hline TBIL ( $\mu \mathrm{mol} /$ liter $)$ & & $12.5(15.7)$ & $8.1(2.1-126.0)$ \\
\hline ALT (U/liter) & & $25.4(18.1)$ & $21.0(5.0-100.0)$ \\
\hline AST (U/liter) & & $37.8(39.3)$ & $26.5(6.0-283.0)$ \\
\hline$\gamma$-GT (U/liter) & & $65.1(84.0)$ & $38.0(7.0-421.0)$ \\
\hline
\end{tabular}

aRange, minimum value-maximum value; $\mathrm{WT}$, current body weight; $\mathrm{Ht}$, height; $\mathrm{BSA}$, body surface area; $\mathrm{BUN}$, blood urea nitrogen; SCR, serum creatinine concentration; UA, uric acid concentration; Cys-C, serum cystatin C concentration; eGFR, estimated glomerular filtration rate; TBIL, total bilirubin concentration; ALT,

alanine aminotransferase concentration; AST, aspartate aminotransferase concentration; $\gamma$-GT,

$\gamma$-glutamyltranspeptidase concentration.

the efficacy of linezolid therapy in adults was correlated with a ratio of the area under the drug plasma concentration-time curve over $24 \mathrm{~h}\left(\mathrm{AUC}_{\mathrm{O}-24}\right)$ to the MIC $\left(\mathrm{AUC}_{\mathrm{O}-24} /\right.$ MIC) of between 80 and 120 (11).

Although several studies have assessed the pharmacokinetics (PKs) of linezolid in pediatric patients, its pharmacokinetic profile has not been fully characterized and the dose regimen needed to achieve a PK/pharmacodynamic (PD) target has yet to be established. Improper dosing in pediatric patients may cause treatment failure, antibiotic resistance, or increased toxicity.

Therefore, the intents of the present study were to evaluate the population pharmacokinetic (PopPK) parameters of intravenous linezolid in children using a modeling and simulation approach and to establish an evidence-based dosing regimen for this vulnerable population.

\section{RESULTS}

Study population. In total, 112 patients were recruited for PopPK analysis from June 2015 to May 2018. Linezolid was well tolerated, and all patients completed their linezolid therapy. The study population consisted of 65 male and 47 female patients with ages ranging from 0.03 to 11.9 years and weights ranging from 2.1 to $46.0 \mathrm{~kg}$. There were 91 children with normal renal function (estimated glomerular filtration rate $\left.[\mathrm{eGFR}] \geq 90 \mathrm{ml} / \mathrm{min} \cdot 1.73 \mathrm{~m}^{2}\right), 17$ children with mild renal insufficiency $(60 \mathrm{ml} /$ $\left.\min \cdot 1.73 \mathrm{~m}^{2} \leq \mathrm{eGFR}<90 \mathrm{ml} / \mathrm{min} \cdot 1.73 \mathrm{~m}^{2}\right)$, and 4 children with moderate renal insufficiency $\left(30 \mathrm{ml} / \mathrm{min} \cdot 1.73 \mathrm{~m}^{2} \leq \mathrm{eGFR}<60 \mathrm{ml} / \mathrm{min} \cdot 1.73 \mathrm{~m}^{2}\right)$ in the study. None of the patients had severe renal insufficiency (eGFR $<30 \mathrm{ml} / \mathrm{min} \cdot 1.73 \mathrm{~m}^{2}$ ). A summary of the demographic and clinical characteristics of the patients is presented in Table 1.

Population pharmacokinetic model development. A total of 135 linezolid concentrations with a range of from 0.4 to $39.0 \mathrm{mg} / \mathrm{liter}$ were obtained for PopPK modeling; 47 of them were trough concentrations, and 5 of them were peak concentrations (at the time point of $0.5 \mathrm{~h}$ after the end of infusion). The other 83 scavenged samples were obtained at the time points between peak and trough concentrations. The linezolid concentration-versus-time profile is shown in Fig. 1. 


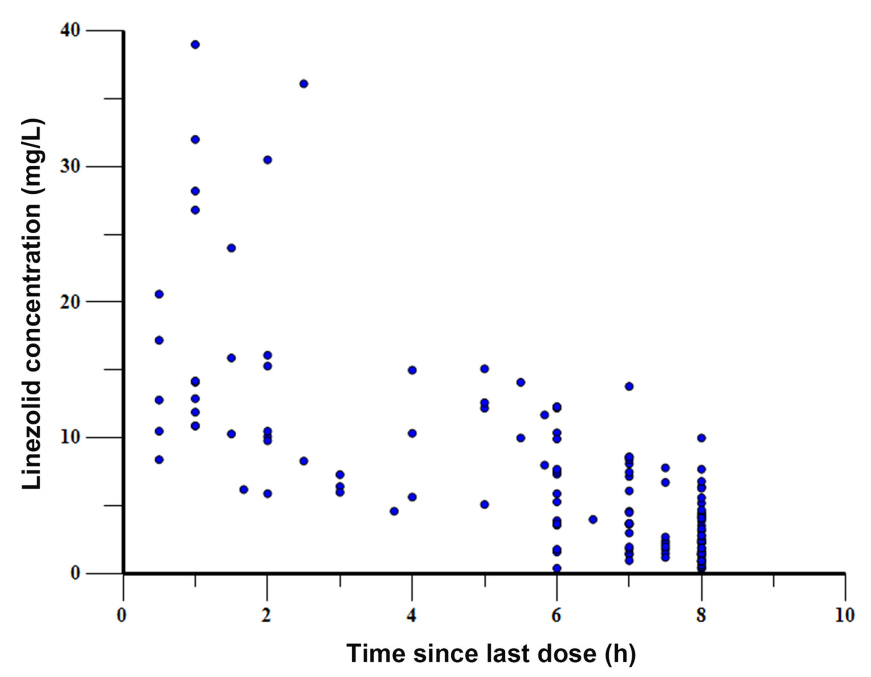

FIG 1 Linezolid concentrations versus time.

The PK characteristics of linezolid could be well illustrated by a one-compartment model with first-order elimination. The one-compartment model resulted in a lower objective function value (OFV) and residual variability than the two-compartment model. The model was parameterized in terms of clearance $(\mathrm{CL})$ and the apparent volume of distribution ( $V$ ). For linezolid, interindividual variability was best described by an exponential model, while residual variability was best fitted by a combined exponential plus additive model.

The result of correlation analysis is displayed in Fig. 2. Under the premise of avoiding the simultaneous incorporation of correlated or colinear variables, the predefined covariates were added to the base model to create a full model. A prior inclusion of current body weight (WT) on clearance and the volume of distribution decreased the OFV by 22.35 points and 16.51 points, respectively. Addition covariate analysis indicated that eGFR exhibited a significant impact on linezolid clearance, with a drop in the OFV of 7.86 points, and a further reduction of 5.30 points in the OFV was obtained by including the alanine aminotransferase concentration (ALT) in the forward inclusion procedure. After incorporating these covariates, no other covariates were found to significantly influence linezolid PKs. In the backward elimination procedure, WT and eGFR were retained in the final model as significant covariates. Details of the covariate screening process are shown in Table 2.

The final PopPK model is represented as follows:

$$
\begin{gathered}
\mathrm{CL}=1.31 \times\left(\frac{\ln \mathrm{WT}}{2.40}\right)^{0.83} \times\left(\frac{\ln \mathrm{eGFR}}{4.89}\right)^{0.60} \\
V=4.24 \times\left(\frac{\ln \mathrm{WT}}{2.40}\right)^{0.86}
\end{gathered}
$$

where $C L$ is the individual clearance (in liters per hour), $V$ is the individual volume of distribution (in liters), WT is body weight (in kilograms), and eGFR is the estimated glomerular filtration rate. The parameter estimates of the final PopPK model are displayed in Table 3.

The typical values of the PK parameters estimated after normalization to a median weight of $11.0 \mathrm{~kg}$ and a median eGFR of $132.6 \mathrm{ml} / \mathrm{min} \cdot 1.73 \mathrm{~m}^{2}$ were as follows: 1.31 liters/h for $C L$ and 4.24 liters for $V$. Mean \pm standard deviation (SD) Bayesian estimates of $C L$ and $V$ were $0.13 \pm 0.08$ liters $/ \mathrm{h} / \mathrm{kg}$ and $0.42 \pm 0.18$ liters/ $\mathrm{kg}$, respectively. The locally weighted scatterplot smoothing (LOWESS) method was used to visualize the relationship between defined covariates and CL (Fig. 3). The LOWESS curves demonstrate that linezolid CL increased allometrically with body weight and eGFR in children. Furthermore, patients were divided into a normal 


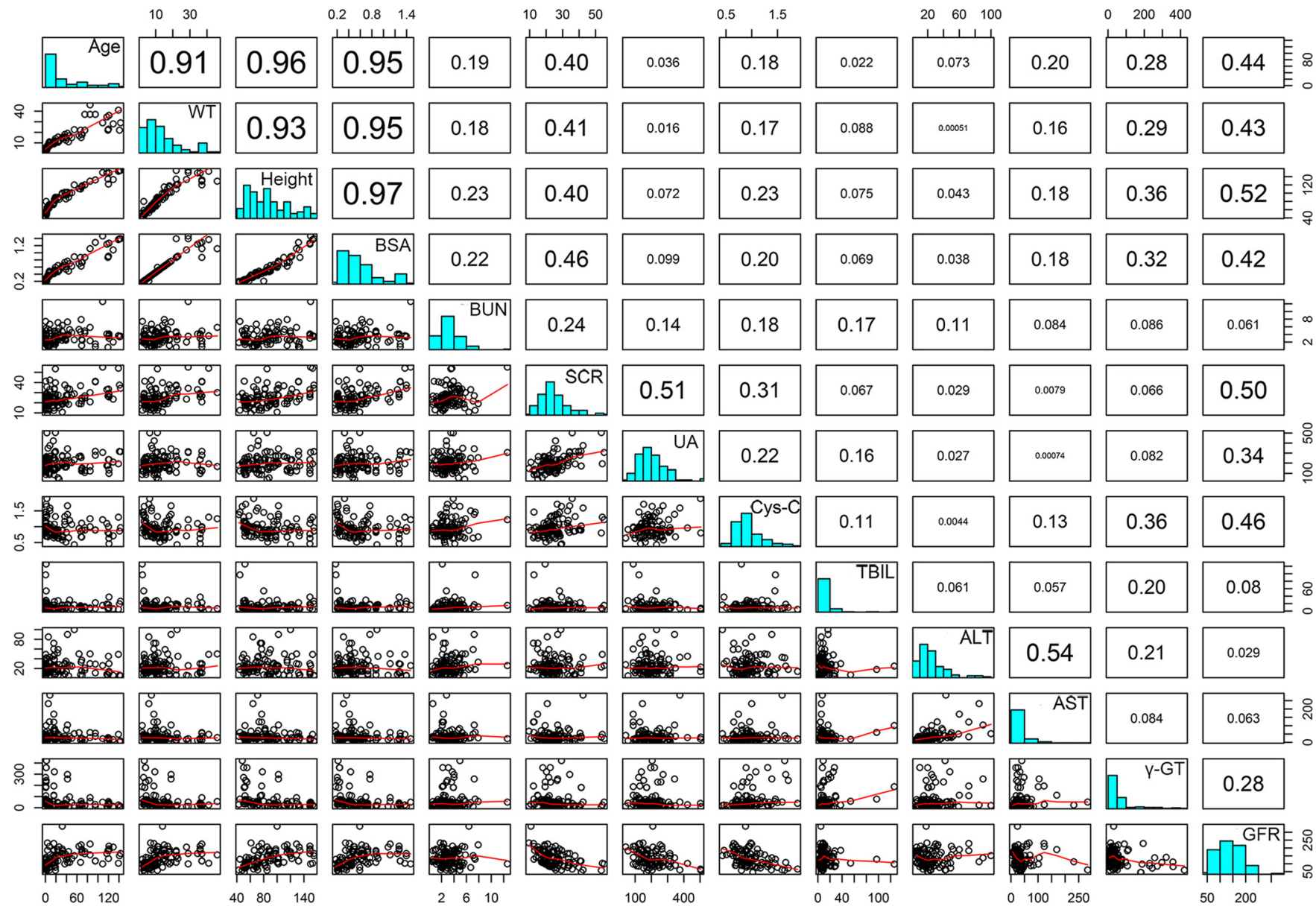

FIG 2 Correlation analysis of covariates. Units of measurement are as follows: age, years; current body weight (WT), kg; height, $\mathrm{cm}$; body surface area (BSA), $\mathrm{m}^{2}$; blood urea nitrogen (BUN), mmol/liter; serum creatinine concentration (SCR), $\mu \mathrm{mol} /$ liter; uric acid (UA), $\mu \mathrm{mol} /$ liter; serum cystatin C (Cys-C), $\mathrm{mg} /$ liter; total bilirubin (TBIL), $\mu \mathrm{mol} /$ liter; alanine aminotransferase (ALT), U/liter; aspartate aminotransferase (AST), U/liter; $\gamma$-glutamyltranspeptidase $(\gamma$-GT), U/liter; estimated glomerular filtration rate (GFR) $\mathrm{ml} / \mathrm{min} \cdot 1.73 \mathrm{~m}^{2}$.

group (eGFR $\geq 90 \mathrm{ml} / \mathrm{min} \cdot 1.73 \mathrm{~m}^{2}$ ) and a renal insufficiency group (eGFR $<90 \mathrm{ml} /$ $\min \cdot 1.73 \mathrm{~m}^{2}$ ) according to the value of eGFR. The result of a $t$ test indicated that the estimated CLs were significantly lower in children with mild to moderate renal insufficiency than in children with normal renal function $(0.75 \pm 0.25$ liters/h versus $1.49 \pm 0.49$ liters $/ \mathrm{h}, P<0.001$ ), but no statistically significant difference in the estimated weight-normalized CLs was observed between the two groups $(0.13 \pm 0.03$ liters $/ \mathrm{h} / \mathrm{kg}$ versus $0.13 \pm 0.09 \mathrm{liters} / \mathrm{h} / \mathrm{kg}, P=0.954$ ).

Model validation. The diagnostic goodness-of-fit plots were visually inspected to confirm the choice of the final model. As presented in Fig. 4, the individual and population predictions were acceptable in terms of visual biases. In the plots of conditional weighted residuals (CWRES) versus population prediction (PRED) and

TABLE 2 Covariate analysis and final model development process ${ }^{a}$

\begin{tabular}{|c|c|c|c|c|c|}
\hline Step & Covariate(s) screened & OFV & $\Delta$ OFV & $P$ value & Comments \\
\hline 1 & None (forward inclusion) & 631.85 & & & Basic model \\
\hline 3 & CL-In WT, $V-\ln W T$ & 592.99 & -16.51 & $<0.01$ & \\
\hline 5 & CL-In WT-In eGFR-ALT, V-In WT (backward elimination) & 579.83 & -5.30 & $<0.05$ & Full model \\
\hline 6 & CL-In WT-In eGFR, V-In WT & 585.13 & 5.30 & $>0.01$ & Final model \\
\hline
\end{tabular}


TABLE 3 Linezolid PopPK parameter estimates from the final model and bootstrap results ${ }^{a}$

\begin{tabular}{|c|c|c|c|c|c|c|}
\hline \multirow[b]{2}{*}{ Parameter } & \multicolumn{2}{|c|}{ Final model } & \multicolumn{3}{|c|}{ Bootstrap analysis } & \multirow[b]{2}{*}{ Bias $(\%$} \\
\hline & Estimate & SE (\%) & 2.5th percentile & Median estimate & 97.5th percentile & \\
\hline$\overline{\theta_{V} \text { (liters) }}$ & 4.24 & 5.7 & 3.80 & 4.23 & 4.77 & -0.24 \\
\hline$\theta_{\mathrm{CL}}\left(\right.$ liters $\left.\cdot \mathrm{h}^{-1}\right)$ & 1.31 & 4.2 & 1.20 & 1.31 & 1.41 & 0 \\
\hline$\theta_{2}$ & 0.83 & 14.3 & 0.45 & 0.82 & 1.19 & -1.21 \\
\hline$\theta_{3}$ & 0.60 & 41.7 & 0.04 & 0.57 & 1.10 & -5.00 \\
\hline$\omega_{\mathrm{CL}}(\%)$ & 39.10 & 4.9 & 35.58 & 41.26 & 46.94 & 5.52 \\
\hline \multicolumn{7}{|l|}{ Residual variability } \\
\hline$\sigma_{\text {pro }}(\%)$ & 16.48 & 17.1 & 7.99 & 15.44 & 22.89 & -6.31 \\
\hline$\sigma_{\text {add }}(\mathrm{mg} /$ liter $)$ & 0.02 & 17.0 & 0.01 & 0.02 & 0.03 & 0 \\
\hline
\end{tabular}

aPopPK, population pharmacokinetics; $\theta_{V}$, typical value of apparent clearance; $\theta_{\mathrm{CL}}$, typical value of apparent volume of distribution; $\theta_{1}$, exponent for In WT as a covariate for $V ; \theta_{2}$, exponent for In WT as a covariate for $C L ; \theta_{3}$, exponent for In eGFR as covariate for $C L ; \omega_{V}$, square root of interindividual variance for $V ; \omega_{C L}$, square root of interindividual variance for $\mathrm{CL} ; \sigma_{\mathrm{pro}}$, residual variability for proportional error; $\sigma_{\text {add }}$, residual variability for additive error; bias $=[($ median estimate by bootstrap analysis - estimate from the final model)/estimate from the final model] $\times 100$.

time, most concentration data were equally distributed around zero and were in the -3 to +3 range. The medians with $95 \%$ confidence interval $(\mathrm{Cl})$ parameter estimates obtained from a 1,000-run bootstrap analysis are given in Table 3 . The parameter estimates of the final PopPK model lay within the $95 \% \mathrm{Cls}$ that resulted
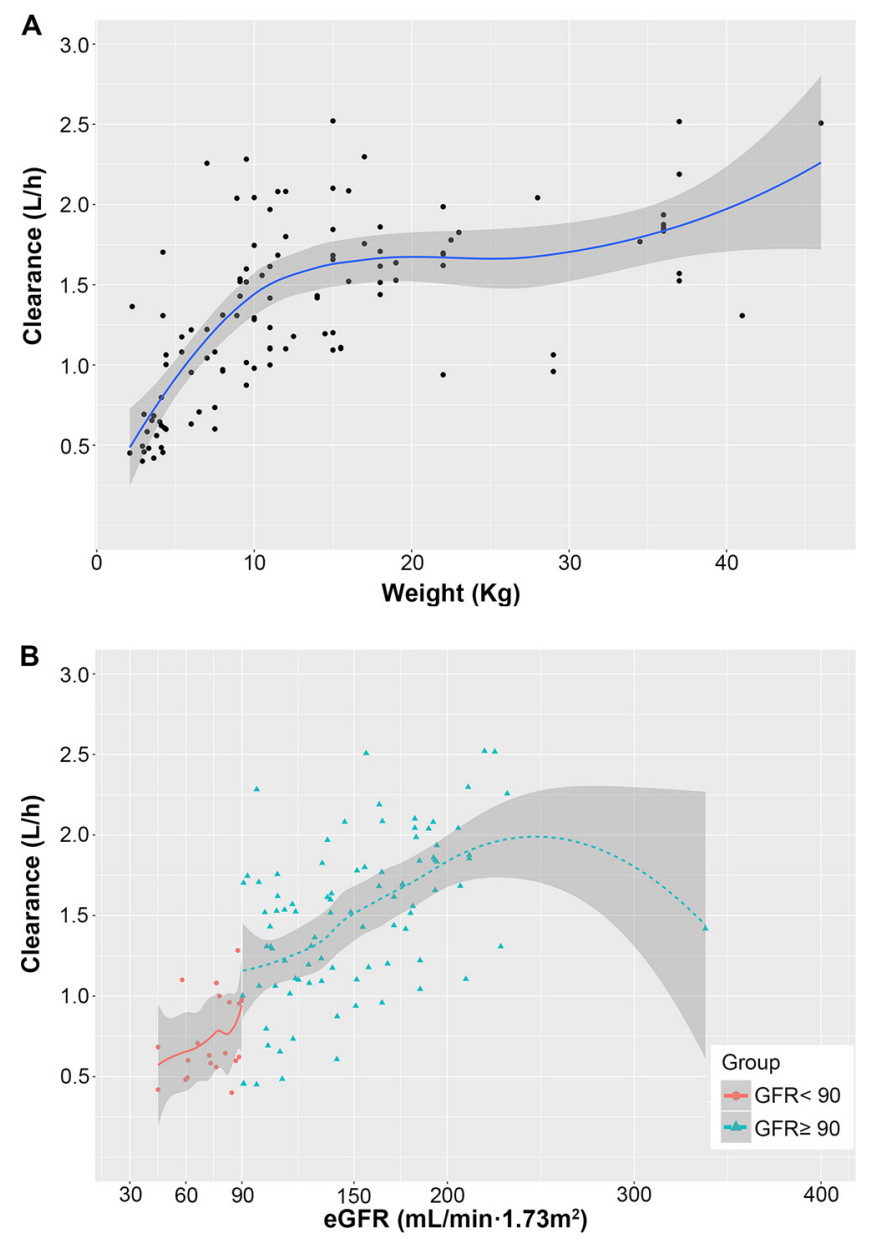

FIG 3 Relationship between linezolid clearance $(C L)$ and body weight $(A)$ and estimated glomerular filtration rate (eGFR) (B). The shaded area represents the $95 \%$ confidence intervals for the locally weighted scatterplot smoothing (LOWESS) fit. 

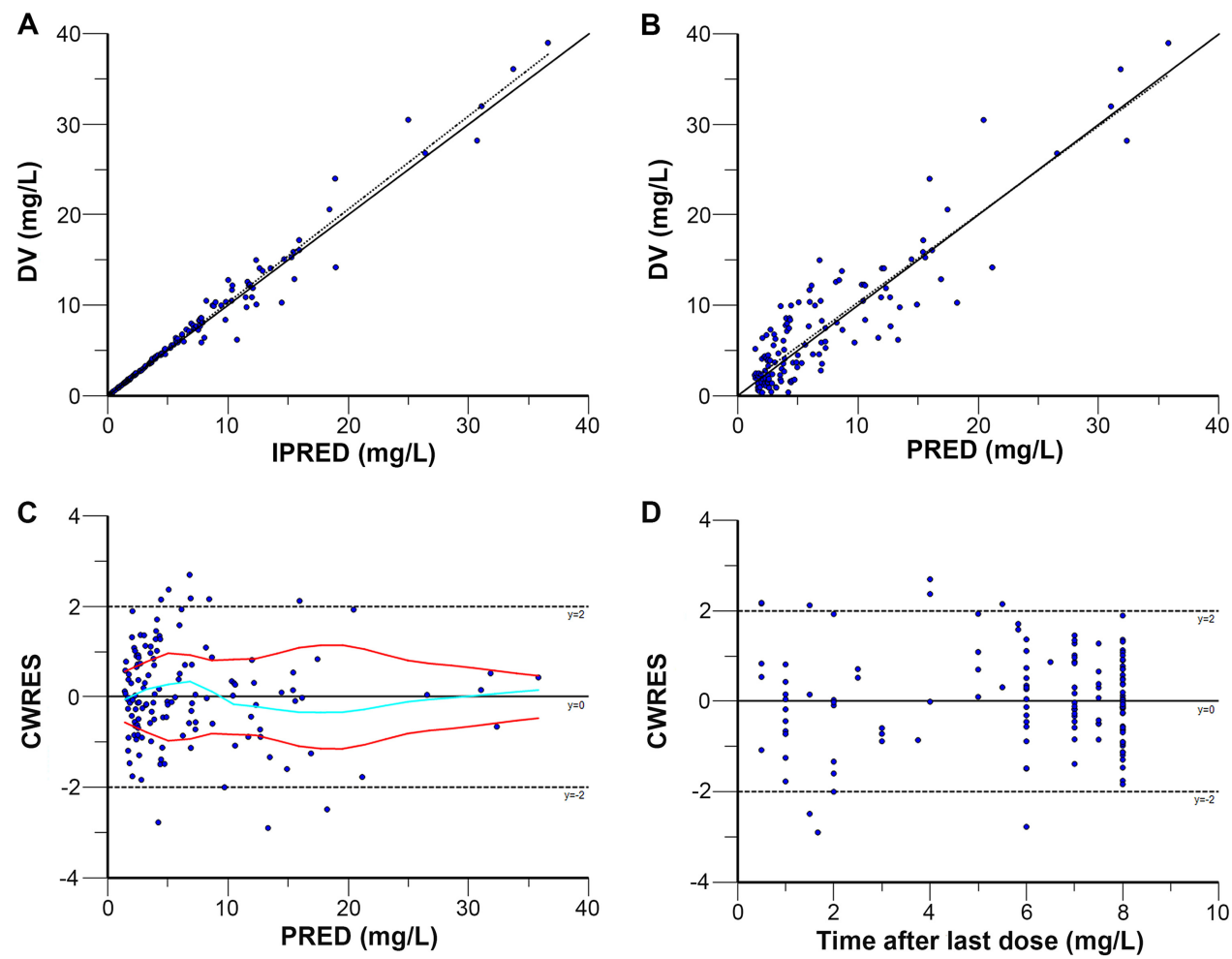

FIG 4 Goodness-of-fit plot for the final model. (A) Observed concentrations (DV) versus individual prediction (IPRED); (B) DV versus population prediction (PRED); (C) conditional weighted residuals (CWRES) versus PRED; (D) CWRES versus time since the last dose.

from the nonparametric bootstrap procedure, and the biases between the final model estimates and the bootstrapped median parameter estimates were less than $\pm 10 \%$ for all parameters, which demonstrated the good stability of the final model. The results of normalized prediction distribution error (NPDE) analysis are displayed in Fig. 5. The NPDEs followed a normal distribution and density, exhibiting a good fit of the final model to the individual data. The $P$ values obtained using the Wilcoxon signed-rank test, Fisher's variance test, Shapiro-Wilks test, and the global test of the normality of the final model were 0.327, 0.184, 0.888, and 0.551, respectively. A visual predictive check (VPC) of concentrations versus time after the last dose is shown in Fig. 6. The majority of the simulations were within the $90 \%$ confidence interval of the prediction, indicating acceptable agreement between observed concentrations and simulated concentrations. Overall, the linezolid PopPK model evaluation results revealed that the final model provided an adequate description of the data and a good prediction of individual PK parameters.

Simulation and dosing regimen optimization. Figure 7 presents the probability of target attainment (PTA; in percent), calculated based on the PopPK model at MIC values in the range of 0.5 to $4 \mathrm{mg} /$ liter for different linezolid dosage regimens. As a result of Monte Carlo simulations, a PTA of $\geq 90 \%$ was achieved with all regimens in pediatric populations for an MIC of $0.5 \mathrm{mg} /$ /iter, whereas all regimens resulted in unacceptably low PTAs at an MIC of $4 \mathrm{mg} /$ liter. At an MIC of $1 \mathrm{mg} /$ liter, dosage regimens of 10 to $20 \mathrm{mg} / \mathrm{kg}$ of body weight every $8 \mathrm{~h}$ (q8h) reached PTAs of $\geq 90 \%$, while a dosage of $5 \mathrm{mg} / \mathrm{kg}$ every $8 \mathrm{~h}$ led to a suboptimal PTA (80.77\%). When considering an MIC of $2 \mathrm{mg} / \mathrm{liter}$, the dosages of $15 \mathrm{mg} / \mathrm{kg}$ and $20 \mathrm{mg} / \mathrm{kg}$ every $8 \mathrm{~h}$ allowed the achievement of nearly optimal PTAs $(94.75 \%$ and $98.36 \%$, respectively). With the recommended dosage regimen prescribed above (10 mg/kg every $8 \mathrm{~h}$ ), only $81.70 \%$ and $35.81 \%$ of the children achieved the pharmacodynamic target $\left(\mathrm{AUC}_{0-24} / \mathrm{MIC} \geq 80\right.$ ) against pathogens with MICs of $2 \mathrm{mg} /$ liter and $4 \mathrm{mg} /$ liter, respectively, indicating that an elevated dosage is required. 

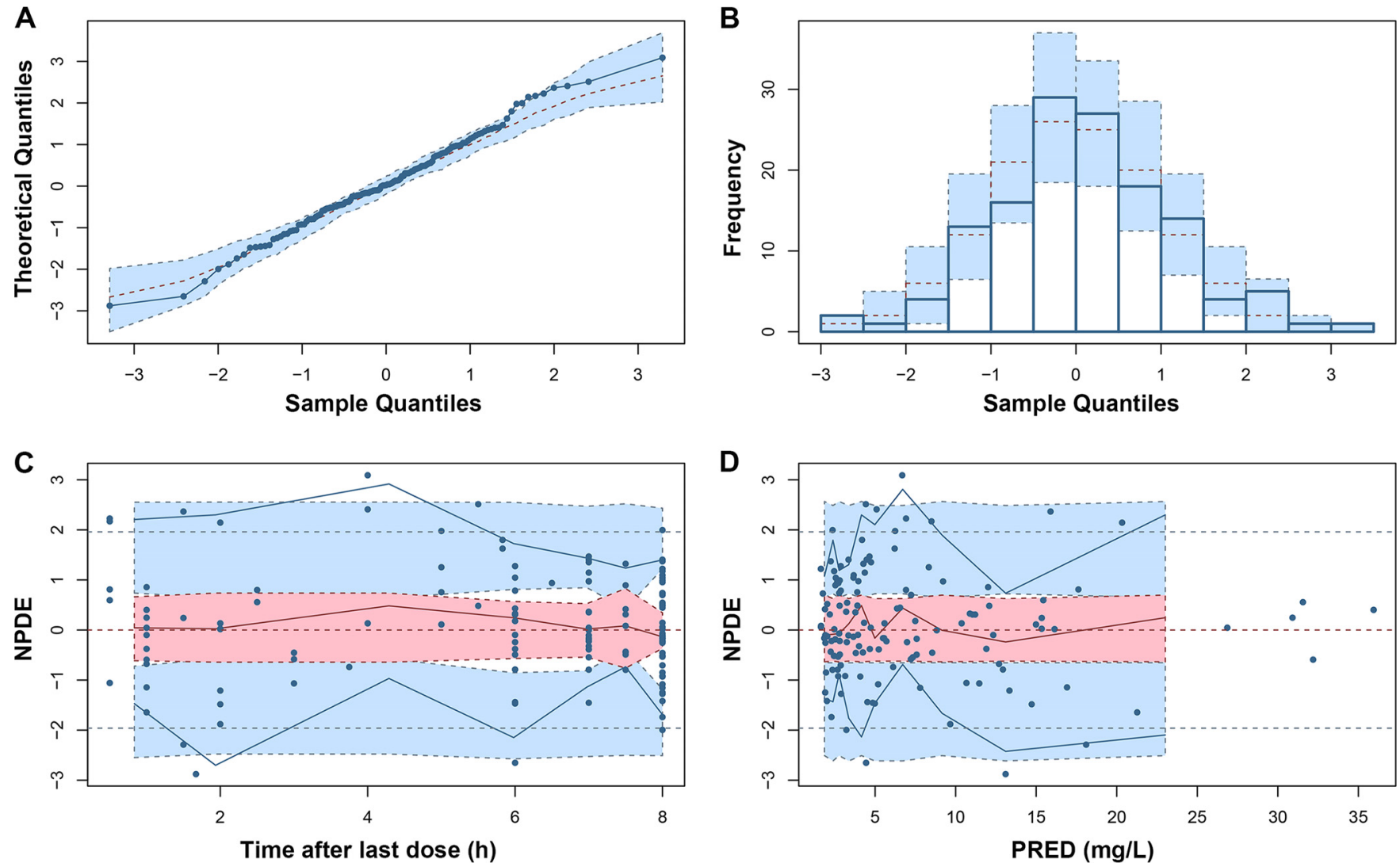

FIG 5 Normalized prediction distribution errors (NPDE) of the final population pharmacokinetic model. (A) Quantile-quantile plot of NPDE versus the expected standard normal distribution; (B) histogram of NPDE with the density of the standard normal distribution overlaid; (C) scatterplot of NPDE versus time after the last dose; (D) scatterplot of NPDE versus population prediction (PRED). Scatterplots show observed concentrations as filled circles, with solid blue lines representing the 5th and 95th percentiles of observed data and red lines representing the median of observed data. Red or blue shaded areas represent the $95 \%$ prediction interval for the respective metric.

\section{DISCUSSION}

Linezolid is currently considered an effective alternative to glycopeptide antibiotics for the therapy of multiresistant Gram-positive bacterial infections in the pediatric inpatient population $(12,13)$. Although the clinical application of linezolid in children has increased progressively over the years (14), data on the linezolid PK profile and therapeutic drug monitoring-guided individualized dosing regimens in pediatric patients are very limited.

The present work is the first study that has reported a PopPK model of intravenous linezolid in children aged 0 to 12 years. The model was established and validated to determine linezolid PK parameters and quantify the influence of demographic and clinical factors on the linezolid disposition. The results of PopPK analysis indicated that a one-compartment model with first-order elimination along with WT and eGFR as significant covariates was optimal for PK data modeling. In the current study, the mean $\mathrm{CL}$ of linezolid in children aged 0 to 12 years was 0.13 liters $/ \mathrm{h} / \mathrm{kg}$, which is consistent with the findings of a prior PK study of linezolid in pediatric patients that reported an average $C L$ value of 0.152 liters $/ \mathrm{h} / \mathrm{kg}$ in 14 children aged 2 to 11 years (15). A comparison of our estimates of PK parameters with those reported in the literature is presented in Table 4 (15-17).

After the covariate screening procedure, only WT and eGFR elucidated significant portions of the variance and were included into the final PopPK model. As a covariate representing body size, WT is generally known to have a significant impact on drug disposition $(18,19)$, especially for pediatric patients. So far, the clearance pathways of linezolid have not been fully illustrated. As approximately $30 \%$ of the linezolid 


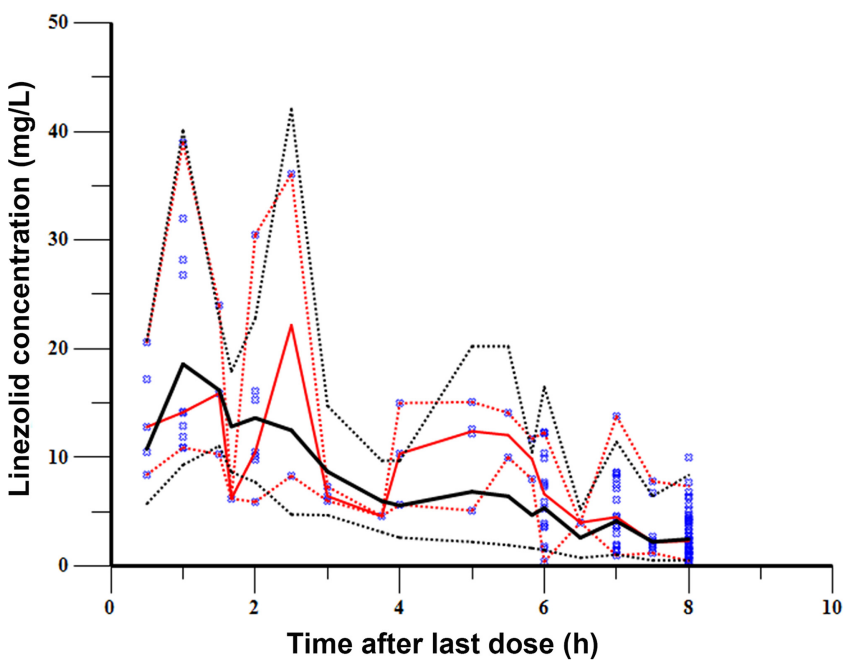

FIG 6 Visual predictive check of the final model. The observed linezolid concentrations are shown as blue circles. Red solid and dashed lines represent the median and the 5th percentile and 95th percentile of the observed concentrations, respectively, and black solid and dashed lines represent the median and the 5th percentile and 95th percentile of the simulated concentrations, respectively.

dose is excreted in urine as the parent compound while $50 \%$ of the administered dose appears in the urine as the two major morpholine ring-oxidized metabolites, linezolid is considered to be substantially renally eliminated (20). Thus, both body size and renal function should have important effect on the dosage regimen in children.

As shown in Fig. 3, CL increased with eGFR, after accounting for body weight variances using an estimated exponent allometric relationship. Consistent with adult data (21), our findings indicate that linezolid clearance is positively correlated with eGFR. The estimated CLs were markedly higher in children with normal renal function than in children with renal insufficiency $(P<0.001)$. However, no statistically significant difference in estimated weight-normalized CLs could be found between the two groups $(P>0.05)$. Therefore, dosing of the medication on a linear weight-adjusted (milligramper-kilogram) basis would be rational for pediatric patients with mild to moderate renal insufficiency or normal renal function (eGFR $\geq 30 \mathrm{ml} / \mathrm{min} \cdot 1.73 \mathrm{~m}^{2}$ ). It should be noted that none of the patients included in our study had severe renal insufficiency (eGFR $<$ $30 \mathrm{ml} / \mathrm{min} \cdot 1.73 \mathrm{~m}^{2}$ ), so we are unable to confirm whether the conclusion made above is appropriate for them.

The results of Monte Carlo simulations demonstrated that the currently recommended pediatric dose of linezolid ( $10 \mathrm{mg} / \mathrm{kg}$ every $8 \mathrm{~h}$ ) would lead to a high risk of underdosing in the target pediatric population when the infection was caused by

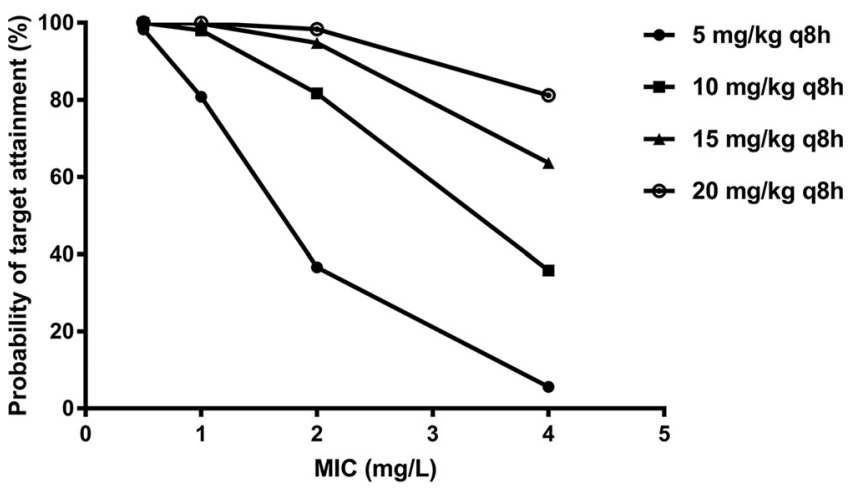

FIG 7 Probability of target $\left(\mathrm{AUC}_{0-24} / \mathrm{MIC} \geq 80\right)$ attainment for different linezolid dosage regimens. 
TABLE 4 Pharmacokinetics of linezolid obtained from different studies ${ }^{a}$

\begin{tabular}{|c|c|c|c|c|c|}
\hline \multirow[b]{3}{*}{ Parameter } & \multicolumn{5}{|c|}{ Value for the group (reference or source) } \\
\hline & \multirow[b]{2}{*}{ Median (range) for neonates (16) } & \multicolumn{4}{|c|}{ Mean for children from: } \\
\hline & & Reference 15 & Reference 15 & Reference 17 & This study \\
\hline Age $(y r)$ & Neonates & $4.9 \pm 2.8$ & $14.9 \pm 1.3$ & $6.4 \pm 3.2$ & $2.9 \pm 3.4$ \\
\hline WT (kg) & $1.423(0.810-3.256)$ & $23.28 \pm 12.74$ & $61.20 \pm 15.56$ & $27.5 \pm 20.4$ & $13.9 \pm 10.2$ \\
\hline CL (liters/h/kg) & $0.17(0.07-0.31)$ & $0.152 \pm 0.074$ & $0.067 \pm 0.04$ & $0.171 \pm 0.068$ & $0.13 \pm 0.08$ \\
\hline
\end{tabular}

${ }^{a} \mathrm{WT}$, current body weight; $\mathrm{CL}$, clearance; $V$, apparent volume of distribution; NA, data are not available.

pathogens with MICs of $2 \mathrm{mg} /$ liter or $4 \mathrm{mg} /$ liter. This is in accordance with the findings of a previous PK/PD study of linezolid in pediatric patients (15), which clarified that recommended dosages were suboptimal in half of the children aged 2 to 11 years old and that higher doses may be needed for them in the presence of bacteria with MICs of $>1 \mathrm{mg} /$ liter. In our study, different linezolid target exposures are required for efficacy according to the MIC of the treating bacteria. In order to reach an adequate drug exposure and the optimal $\mathrm{AUC}_{0-24} / \mathrm{MIC}$ ratio, an elevated dosage of 15 or $20 \mathrm{mg} / \mathrm{kg}$ every $8 \mathrm{~h}$ is necessary for children infected with Gram-positive bacteria with higher linezolid MICs of $\geq 2 \mathrm{mg} /$ liter. It is noteworthy that some studies have revealed a correlation between high linezolid exposure and the induction of hematotoxicity (e.g., thrombocytopenia and anemia) (21-24). As previously reported in adults (25), overexposure was defined as an $A_{U C} C_{0-24}$ of $\geq 300 \mathrm{mg} \cdot \mathrm{h} / \mathrm{liter}$. Simulation showed that the probability of attainment of the $\mathrm{AUC}_{0-24}$ target value for the maximum dose of $20 \mathrm{mg} / \mathrm{kg}$ reached up to $83.95 \%$, which implied an increased risk of drug-related hematotoxicity. In such cases, other antibacterial agents should be considered. However, if there is no other choice, a daily dose of linezolid of up to $60 \mathrm{mg} / \mathrm{kg} / \mathrm{day}$ could be considered to achieve PK/PD targets, provided that clinical symptoms and hemogram parameters are monitored closely during the treatment to avoid linezolid-related overexposure and toxicity.

Our study had several limitations. The final model of linezolid was only internally validated; external validation was not implemented due to the limited number of patients. In addition, the efficacy and safety of linezolid treatment were not evaluated in the current study owing to the scarcity of clinical data.

Conclusion. In this study, we characterized the PKs of linezolid in pediatric patients aged 0 to 12 years and assessed the impact of demographic characteristics and clinical covariates on the linezolid disposition. Only WT and eGFR were found to be determinants of linezolid clearance, and the volume of distribution was influenced by WT in our PopPK analysis. For pediatric patients infected with pathogens with an MIC of $\geq 2 \mathrm{mg} /$ liter, the currently recommended dose regimen of $10 \mathrm{mg} / \mathrm{kg}$ q $8 \mathrm{~h}$ may be inadequate and a higher dose of 15 to $20 \mathrm{mg} / \mathrm{kg}$ q8h would be required to achieve the PK/PD target and ensure the efficacy of linezolid therapy. The final PopPK model could be utilized to predict the individual PK parameters for a given patient treated with intravenous linezolid and provide an evidence-based approach for linezolid dosage individualization in the pediatric population.

\section{MATERIALS AND METHODS}

Study design. A prospective, open-label PK study of linezolid was conducted at Wuhan Children's Hospital from June 2015 to May 2018. The inclusion criteria were as follows: pediatric patients aged 0 to 12 years with confirmed or suspected multiresistant Gram-positive bacterial infections receiving the same dose of linezolid intravenously for at least 3 days. The exclusion criteria were as follows: patients enrolled in another clinical trial, patients who were intolerant to linezolid treatment, and patients with other factors that the researchers considered made them unsuitable for inclusion.

This study was designed in accordance with legal requirements and the Declaration of Helsinki and was approved by the Ethics Committee of Wuhan Children's Hospital. Written informed consent was obtained from the parents or guardians of the patients. 
Dosage regime and pharmacokinetic sampling. Linezolid for injection (Zyvox; Pfizer Inc.) was administered as a 1-h intravenous infusion at a dose of $10 \mathrm{mg} / \mathrm{kg}$ three times a day. An opportunistic sampling strategy was performed when a steady-state linezolid concentration had been achieved (at least 3 days from the start of treatment) (26). Residual blood samples were collected from specimens required for routine biochemical tests. Plasma was separated by centrifugation for $5 \mathrm{~min}$ at 15,000 rpm immediately before it was stored at $-80^{\circ} \mathrm{C}$. Plasma concentrations were determined within $72 \mathrm{~h}$ after sampling. The precise dosing and infusion time as well as the sampling time were recorded prospectively. Individual laboratory parameters before linezolid treatment and demographic data for the patients were collected through an electronic medical record database in the hospital and included age, gender, current body weight (WT), height, body surface area (BSA), blood urea nitrogen concentration (BUN), serum creatinine concentration (SCR), uric acid concentration (UA), serum cystatin C concentration (Cys-C), total bilirubin concentration (TBIL), alanine aminotransferase concentration (ALT), aspartate aminotransferase concentration (AST), and $\gamma$-glutamyltranspeptidase concentration ( $\gamma$-GT). The estimated glomerular filtration rate (eGFR) was calculated according to the modified formula of Schwartz et al. (27).

Analytical method of linezolid. Linezolid concentrations were determined using a validated high-performance liquid chromatography (HPLC) method (1260 Infinity column; Agilent Technologies Inc.) with UV detection. For sample preparation, $0.5 \mathrm{ml}$ of serum was loaded onto a solid-phase extraction column $\left(200 \mathrm{mg} / 3 \mathrm{ml}\right.$; Cleanert ODS $\mathrm{C}_{18}$; Agela Technologies) and eluted with $30 \%$ acetonitrile. Separation was achieved by using an Innoval $C_{18}$ column (particle size, $10 \mu \mathrm{m} ; 100 \AA ̊$; 4.6 by $250 \mathrm{~mm}$; Agela Technologies). A solution of $25 \%$ acetonitrile was used as the mobile phase. The pump flow rate was $0.8 \mathrm{ml} / \mathrm{min}$, and the column temperature was $30^{\circ} \mathrm{C}$. The wavelength of optimum UV detection was set at $254 \mathrm{~nm}$. The calibration curve was linear over a concentration range of 0.4 to $50 \mathrm{mg} / \mathrm{liter}$. The intra- and interassay coefficients of variation were less than $10 \%$. The lower limit of quantification (LLOQ) was $0.4 \mathrm{mg} / \mathrm{liter}$.

Population pharmacokinetic modeling of linezolid. PopPK analysis was performed using the nonlinear mixed-effects modeling program NONMEM (version 7.3; Icon Inc., PA, USA). The R program (version 3.5.1; https://www.r-project.org/) was utilized to construct the output visualizations and the model validations. The first-order conditional estimation-extended least-squares method (FOCE-ELS) was used throughout the model development procedure.

Base model. The PopPK model consists of a structural model that illustrates the concentration-time relationship and random effect models that describe the inter- and intraindividual variability of the PKs. One- and two-compartment structural models with first-order elimination were evaluated on the basis of previously reported PK models and exploratory graphical analysis $(15,16)$. The initial structural model was selected according to visual inspection of routine diagnostic plots and various goodness-of-fit criteria, including precision and the plausibility of parameter estimation, improvement of the objective function value (OFV), the Akaike information criterion (AIC), and the Bayesian information criterion (BIC). $\mathrm{OFV}$ is defined as the value of $-2 \times \log$ likelihood in the maximum likelihood approach. AIC and BIC are defined as follows:

$$
\begin{gathered}
\mathrm{AIC}=\mathrm{OFV}+2 p \\
\mathrm{BIC}=\mathrm{OFV}+p \ln n
\end{gathered}
$$

where $p$ is the number of parameters in the model, and $n$ is the sample size. For both indexes, smaller values indicate better models.

The interindividual variability of the PK parameters was described by an exponential model (equation 5).

$$
P_{i}=\theta \times \exp \left(\eta_{i}\right)
$$

where $P_{i}$ represents the estimated parameter value for individual $I, \theta$ is the population typical value of the parameter, and $\eta_{i}$ is a random variable for individual $i$, which is assumed to follow a normal distribution with a mean of zero and a variance of $\omega^{2}$.

The intraindividual variability (residual error) was evaluated by a combined additive and proportional model (equation 6).

$$
Y=\operatorname{IPRED} \times\left(1+\varepsilon_{1}\right)+\varepsilon_{2}
$$

where $Y$ represents the measured observation, IPRED is the individual prediction, and $\varepsilon_{1}$ and $\varepsilon_{2}$ are proportional and additive residual random errors, respectively, which are assumed to follow a Gaussian distribution with a mean of zero and a variance of $\sigma^{2}$.

Covariate analysis. Covariate analysis followed a stepwise forward inclusion and backward elimination procedure, and the likelihood ratio test was used to assess the effect of each covariate on the model parameters. Demographic characteristics (including age, gender, WT, height, BSA), renal functions (BUN, SCR, UA, Cys-C, eGFR), and hepatic functions (TBIL, ALT, AST, $\gamma$-GT) were investigated as potential variables on PK parameters. Correlation analysis was performed to ensure that the final model would not simultaneously include the effects of correlated or colinear variables. The effects of continuous covariates were modeled using a median-normalized power model, while the effects of categorical covariates were described by an exponential model (equations 7 and 8 ).

$$
\begin{gathered}
\theta_{i}=\theta \times\left(\frac{\operatorname{cov}_{j}}{\operatorname{cov}_{\text {median }}}\right)^{\theta_{\text {cov }}} \\
\theta_{i}=\theta \times \exp \left(\theta_{\text {cov }}\right)
\end{gathered}
$$


where $\operatorname{cov}_{j}$ represents the jth covariate, $\operatorname{cov}_{\text {median }}$ is the median of the covariate, $\theta_{i}$ is the ith population prediction of the parameter, $\theta$ is the population typical value of the parameter, and $\theta_{\text {cov }}$ is an estimated parameter describing the fixed effect of the covariate on the PK parameter.

During the stepwise forward inclusion step, a covariate was considered significant and included in the basic model if the OFV decreased by more than 3.84 points with a $P$ value of $<0.05$ ( $\chi^{2}$ distribution with 1 degree of freedom). All of the significant covariates were then incorporated into the basic model to construct a full model. Next, backward elimination was used to exclude covariates from the full model with an increase in the OFV of $<6.635$ ( $\chi^{2}$ distribution with 1 degree of freedom) at the statistical level of a $P$ value of $<0.01$ (28). The resulting model was considered the final PopPK model. The individual parameter estimates were derived from the final model by a post hoc empirical Bayesian method, and the value of $\mathrm{AUC}_{0-24}$ was calculated according to the formula $\mathrm{AUC}_{0-24}=$ daily dose/clearance.

Model validation. Goodness-of-fit plots, nonparametric bootstrap analysis $(29,30)$, normalized prediction distribution errors (NPDEs) (31), and a visual predictive check (VPC) (32) were employed to evaluate the final model and parameter estimates. Goodness-of-fit plots, including observed concentrations (DV) versus individual prediction (IPRED), DV versus population prediction (PRED), conditional weighted residuals (CWRES) versus PRED, and CWRES versus time, were initially used for diagnostic purposes (33). Then a nonparametric bootstrap procedure was conducted to assess the performance and stability of the final model. Random sampling with replacement was utilized to generate 1,000 replicate data sets using the individual as the sampling unit. The final PopPK model was fitted to each of these bootstrap data sets, and all model parameters were estimated. The median and $95 \%$ confidence intervals $(\mathrm{Cls})$ of the resulting parameters were calculated and compared with the final parameter estimates obtained using the NONMEN program. In order to implement the VPC method and NPDE evaluation, simulation replicates of the original data set were performed 1,000 times by using the final PopPK model, and the observations were compared with the distribution of simulated data. The NPDE results were summarized graphically by the add-on package for $\mathrm{R}(31)$, including a quantile-quantile plot, a histogram of the NPDE distribution, and plots of NPDE versus PRED and versus time after dose. The NPDE is assumed to follow a normal distribution. For the VPC, the $95 \% \mathrm{Cls}$ for the 5th, 50th, and 95th percentiles of the simulated concentrations were calculated, plotted against time after dose, and compared with the observed concentrations.

Simulation and dosing regimen optimization. Monte Carlo simulation was performed by utilizing the parameter estimates obtained from the final PopPK model to explore the optimal dosage regimen. According to the data from the European Committee on Antimicrobial Susceptibility Testing (EUCAST) (34), Staphylococcus aureus and Enterococcus faecium are sensitive to linezolid with MICs of $\leq 4 \mathrm{mg} /$ liter. A 10,000-subject simulation was carried out for three different dosing regimens $(10 \mathrm{mg} / \mathrm{kg}$ every $8 \mathrm{~h}$, $15 \mathrm{mg} / \mathrm{kg}$ every $8 \mathrm{~h}$, and $20 \mathrm{mg} / \mathrm{kg}$ every $8 \mathrm{~h}$ ) in children for each of the flowing MICs: $0.5,1,2$, and $4 \mathrm{mg} /$ liter. During each simulation, a set of clearances for patients with normal or impaired renal function (eGFR $\geq 30 \mathrm{ml} / \mathrm{min} \cdot 1.73 \mathrm{~m}^{2}$ ) was generated on the basis of each mean and standard deviation of the PopPK parameters, and then $\mathrm{AUC}_{0-24}$ values were calculated for each patient simulation. For the assessment of efficacy, the probability of target attainment (PTA) of an $\mathrm{AUC}_{0-24} / \mathrm{MIC}$ ratio threshold of $\geq 80$ was computed. Optimal dosing regimens were chosen according to their capacity to attain a PTA of $\geq 90 \%$ for susceptible pathogens with different MICs.

\section{ACKNOWLEDGMENTS}

This study was supported by the Youth Program of the National Natural Science Foundation of China (grant agreement number 81600123) and the Youth Foundation of the Wuhan Municipal Population and Family Planning Commission (grant agreement number WX17Z13).

We thank all the patients and their families for participating in our study.

We declare no conflict of interest related to this work.

\section{REFERENCES}

1. Zurenko GE, Gibson JK, Shinabarger DL, Aristoff PA, Ford CW, Tarpley WG. 2001. Oxazolidinones: a new class of antibacterials. Curr Opin Pharmacol 1:470-476.

2. Shinabarger D. 1999. Mechanism of action of the oxazolidinone antibacterial agents. Expert Opin Investig Drugs 8:1195-1202. https://doi.org/ 10.1517/13543784.8.8.1195.

3. Hashemian SMR, Farhadi T, Ganjparvar M. 2018. Linezolid: a review of its properties, function, and use in critical care. Drug Des Devel Ther 12:1759-1767. https://doi.org/10.2147/DDDT.S164515.

4. Jungbluth GL, Welshman IR, Hopkins NK. 2003. Linezolid pharmacokinetics in pediatric patients: an overview. Pediatr Infect Dis J 22:S153. https://doi.org/10.1097/01.inf.0000086954.43010.63.

5. Douros A, Grabowski K, Stahlmann R. 2015. Drug-drug interactions and safety of linezolid, tedizolid, and other oxazolidinones. Expert
Opin Drug Metab Toxicol 11:1849-1859. https://doi.org/10.1517/ 17425255.2015.1098617.

6. Ament PW, Jamshed N, Horne JP. 2002. Linezolid: its role in the treatment of gram-positive, drug-resistant bacterial infections. Am Fam Physician 65:663-670.

7. Welshman IR, Sisson TA, Jungbluth GL, Stalker DJ, Hopkins NK. 2001. Linezolid absolute bioavailability and the effect of food on oral bioavailability. Biopharm Drug Dispos 22:91-97.

8. Meagher AK, Forrest A, Rayner CR, Birmingham MC, Schentag JJ. 2003. Population pharmacokinetics of linezolid in patients treated in a compassionate-use program. Antimicrob Agents Chemother 47: 548-553. https://doi.org/10.1128/AAC.47.2.548-553.2003.

9. MacGowan AP. 2003. Pharmacokinetic and pharmacodynamic profile of linezolid in healthy volunteers and patients with Gram-positive infec- 
tions. J Antimicrob Chemother 51(Suppl 2):ii17-ii25. https://doi.org/10 .1093/jac/dkg248.

10. Slatter JG, Stalker DJ, Feenstra KL, Welshman IR, Bruss JB, Sams JP, Johnson MG, Sanders PE, Hauer MJ, Fagerness PE, Stryd RP, Peng GW, Shobe EM. 2001. Pharmacokinetics, metabolism, and excretion of linezolid following an oral dose of $[(14) \mathrm{C}]$ linezolid to healthy human subjects. Drug Metab Dispos 29:1136-1145.

11. Rayner CR, Forrest A, Meagher AK, Birmingham MC, Schentag JJ. 2003. Clinical pharmacodynamics of linezolid in seriously ill patients treated in a compassionate use programme. Clin Pharmacokinet 42:1411-1423. https://doi.org/10.2165/00003088-200342150-00007.

12. Jantausch BA, Deville J, Adler S, Morfin MR, Lopez P, Edge-Padbury B, Naberhuis-Stehouwer S, Bruss JB. 2003. Linezolid for the treatment of children with bacteremia or nosocomial pneumonia caused by resistant gram-positive bacterial pathogens. Pediatr Infect Dis J 22(9 Suppl): S164-S171.

13. Kaplan SL, Deville JG, Yogev R, Morfin MR, Wu E, Adler S, Edge-Padbury B, Naberhuis-Stehouwer S, Bruss JB, Linezolid Pediatric Study Group. 2003. Linezolid versus vancomycin for treatment of resistant Grampositive infections in children. Pediatr Infect Dis J 22:677-686. https:// doi.org/10.1097/01.inf.0000078160.29072.42.

14. Hersh AL, Lee BR, Hedican EB, Herigon JC, Newland JG. 2014. Linezolid use in hospitalized children. Pediatr Infect Dis J 33:e14-e18. https://doi .org/10.1097/INF.0000000000000011.

15. Cojutti P, Maximova N, Crichiutti G, Isola M, Pea F. 2015. Pharmacokinetic pharmacodynamic evaluation of linezolid in hospitalized paediatric patients: a step toward dose optimization by means of therapeutic drug monitoring and Monte Carlo simulation. J Antimicrob Chemother 70: 198-206. https://doi.org/10.1093/jac/dku337.

16. Thibault C, Kassir N, Goyer I, Theoret Y, Litalien C, Moussa A, Ovetchkine $P$, Autmizguine J. 2019. Population pharmacokinetics of intravenous linezolid in premature infants. Pediatr Infect Dis J 38:82-88. https://doi .org/10.1097/inf.0000000000002067.

17. Matsumoto K, Shigemi A, Takeshita A, Watanabe E, Yokoyama Y, Ikawa K, Morikawa N, Takeda Y. 2015. Linezolid dosage in pediatric patients based on pharmacokinetics and pharmacodynamics. J Infect Chemother 21:70-73. https://doi.org/10.1016/j.jiac.2014.08.017.

18. Guk J, Chae D, Park K. 2017. Relationship between body weight and postmenstrual age in a Korean pediatric population. Transl Clin Pharmacol 25:101-105. https://doi.org/10.12793/tcp.2017.25.2.101.

19. Abe S, Chiba K, Cirincione B, Grasela TH, Ito K, Suwa T. 2009. Population pharmacokinetic analysis of linezolid in patients with infectious disease: application to lower body weight and elderly patients. J Clin Pharmacol 49:1071-1078. https://doi.org/10.1177/0091270009337947.

20. Duan P, Fisher JW, Yoshida K, Zhang L, Burckart GJ, Wang J. 2017. Physiologically based pharmacokinetic prediction of linezolid and emtricitabine in neonates and infants. Clin Pharmacokinet 56:383-394. https://doi.org/10.1007/s40262-016-0445-9.

21. Matsumoto K, Takeshita A, Ikawa K, Shigemi A, Yaji K, Shimodozono Y, Morikawa N, Takeda Y, Yamada K. 2010. Higher linezolid exposure and higher frequency of thrombocytopenia in patients with renal dysfunc- tion. Int J Antimicrob Agents 36:179-181. https://doi.org/10.1016/j .ijantimicag.2010.02.019.

22. Tsuji Y, Hiraki Y, Matsumoto K, Mizoguchi A, Kobayashi T, Sadoh S, Morita K, Kamimura H, Karube Y. 2011. Thrombocytopenia and anemia caused by a persistent high linezolid concentration in patients with renal dysfunction. J Infect Chemother 17:70-75. https://doi.org/10.1007/s10156 -010-0080-6.

23. Cattaneo D, Orlando G, Cozzi V, Cordier L, Baldelli S, Merli S, Fucile S, Gulisano C, Rizzardini G, Clementi E. 2013. Linezolid plasma concentrations and occurrence of drug-related haematological toxicity in patients with gram-positive infections. Int J Antimicrob Agents 41:586-589. https://doi.org/10.1016/j.ijantimicag.2013.02.020.

24. Dong HY, Xie J, Chen LH, Wang TT, Zhao YR, Dong YL. 2014. Therapeutic drug monitoring and receiver operating characteristic curve prediction may reduce the development of linezolid-associated thrombocytopenia in critically ill patients. Eur J Clin Microbiol Infect Dis 33:1029-1035. https://doi.org/10.1007/s10096-013-2041-3.

25. Pea F, Viale P, Cojutti P, Del Pin B, Zamparini E, Furlanut M. 2012. Therapeutic drug monitoring may improve safety outcomes of longterm treatment with linezolid in adult patients. J Antimicrob Chemother 67:2034-2042. https://doi.org/10.1093/jac/dks153.

26. Leroux S, Turner MA, Guellec CB, Hill H, van den Anker JN, Kearns GL, Jacqz-Aigrain E, Zhao W. 2015. Pharmacokinetic studies in neonates: the utility of an opportunistic sampling design. Clin Pharmacokinet 54: 1273-1285. https://doi.org/10.1007/s40262-015-0291-1.

27. Schwartz GJ, Muñoz A, Schneider MF, Mak RH, Kaskel F, Warady BA, Furth SL. 2009. New equations to estimate GFR in children with CKD. J Am Soc Nephrol 20:629-637. https://doi.org/10.1681/ASN.2008030287.

28. Jonsson EN, Karlsson MO. 1998. Automated covariate model building within NONMEM. Pharm Res 15:1463-1468.

29. Ette El, Onyiah LC. 2002. Estimating inestimable standard errors in population pharmacokinetic studies: the bootstrap with Winsorization. Eur J Drug Metab Pharmacokinet 27:213-224. https://doi.org/10.1007/ BF03190460.

30. Ette El, Williams PJ, Kim YH, Lane JR, Liu MJ, Capparelli EV. 2003. Model appropriateness and population pharmacokinetic modeling. J Clin Pharmacol 43:610-623.

31. Comets E, Brendel K, Mentre F. 2008. Computing normalised prediction distribution errors to evaluate nonlinear mixed-effect models: the NPDE add-on package for R. Comput Methods Programs Biomed 90:154-166. https://doi.org/10.1016/j.cmpb.2007.12.002.

32. Bergstrand M, Hooker AC, Wallin JE, Karlsson MO. 2011. Predictioncorrected visual predictive checks for diagnosing nonlinear mixedeffects models. AAPS J 13:143-151. https://doi.org/10.1208/s12248-011 -9255-z.

33. Hooker AC, Staatz CE, Karlsson MO. 2007. Conditional weighted residuals (CWRES): a model diagnostic for the FOCE method. Pharm Res 24: 2187-2197. https://doi.org/10.1007/s11095-007-9361-x.

34. EUCAST. 2018. Breakpoint tables for interpretation of MICs and zone diameters. EUCAST, Växjö, Sweden. 